\title{
Bayesian Inference on the Generalized Gamma Distribution Based on Generalized Order Statistics
}

\author{
M. Maswadah ${ }^{1}$, Ali M. Seham ${ }^{2}$ and M. Ahsanullah ${ }^{3}$ \\ Received 11 February 2013 \\ Accepted 7 August 2013
}

\begin{abstract}
In this paper, the confidence intervals for the generalized gamma distribution parameters are derived based on the Bayesian approach using the informative and non-informative priors and the classical approach, via the Asymptotic Maximum likelihood estimation, based on the generalized order statistics. For measuring the performance of the Bayesian approach comparing to the classical approach, the confidence intervals of the unknown parameters have been studied, via Monte Carlo simulations and some real data. The simulation results indicated that the confidence intervals based on the Bayesian approach compete and outperform those based on the classical approach.
\end{abstract}

Keywords: Generalized gamma distribution; Generalized order statistics; Asymptotic maximum likelihood estimation; Bayesian inference.

\footnotetext{
${ }_{1,2}$ Department of Mathematics, Faculty of science

Aswan University, Aswan, Egypt

E-mail: maswadah@hotmail.com

E-mail: seham_1elwany@yahoo.com

${ }^{3}$ Department of Management Sciences, Rider University

Lawrenceville, NJ 08648-3099, USA

E-mail:ahsan@rider.edu
} 


\section{Introduction}

A random variable $X$ is said to have generalized gamma distribution (GGD) if its probability density function (PDF) has the form:

$f(x ; \alpha, \beta, \lambda)=\frac{\alpha}{\Gamma(\lambda)} \beta^{-\alpha \lambda} x^{\alpha \lambda-1} \exp \left[-(x / \beta)^{\alpha}\right], \quad x>0 \alpha, \beta, \lambda>0$.

$\Gamma(\lambda)$ is the gamma function, $\alpha, \beta$ and $\lambda$ are the shape, scale and index (or shape) parameters respectively. The corresponding cumulative distribution function (CDF) is given by:

$$
F(x ; \alpha, \beta, \lambda)=\frac{I G\left(\lambda,(x / \beta)^{\alpha}\right)}{\Gamma(\lambda)}, \quad x>0,
$$

where

$$
I G\left(\lambda,(x / \beta)^{\alpha}\right)=\int_{0}^{(x / \beta)^{\alpha}} t^{\lambda-1} e^{-t} d t \text {, is the lower incomplete gamma }
$$

function.

Generalized gamma distribution has been proposed by Stacy (1962), as a flexible family, that includes some important lifetime models as special cases by setting the shape parameters to unity, such as, the exponential distribution $(\lambda=\alpha=1)$, gamma distribution $(\alpha=1)$, Weibull distribution $(\lambda=1)$ and lognormal distribution as $\lambda \rightarrow \infty$.

The inferential procedures for the GGD are difficult perhaps because of an additional shape parameter. However, the statistical analysis of its parameters based on complete as well as censored samples have been studied by many authors such as, Stacy and Mihram (1965), Harter ( 1967), Hager and Bain (1970), Prentice (1974), Lawless (1980, 1982), Di Ciccio (1987), Wingo (1987), Cohen and Whitten (1988), Maswadah (1989, 1991), Hwang and Hu (1999), Hwang and Huang (2006), Gomes 
et al. (2008), Geng and Yuhlong (2009). For Bayesian inference, one may refer to Dadpay et al. (2007) and Mukherjee et al. (2011).

Up to now, Bayesian and non-Bayesian estimation related to the GGD based on the generalized order statistics (GOS) were not addressed in the literature. Thus, in this paper, the Bayesian inference using the informative and non-informative priors are derived based on the GOS, that introduced by Kamps (1995) as a unified approach to several models of ordered random variables such as ordinary order statistics, type-II censored order statistics, type-II progressive censored order statistics, upper record values and sequential order statistics. For more details about the GOS, see among others Ahsanullah (1995, 2000).

Let $X(1, n, \tilde{m}, k), \ldots, X(n, n, \tilde{m}, k),(k \geq 1, \tilde{m}>-1$ is a real number) be $\mathrm{n}$ generalized order statistics from a continuous population with $\mathrm{CDF} F(x)$ and PDF $f(x)$, thus their joint PDF has the form

$$
\begin{array}{r}
f_{X(1, n, \tilde{m}, k), \ldots, X(n, n, \tilde{m}, k)}\left(x_{1}, x_{2}, \ldots, x_{n}\right)=k \prod_{i=1}^{n-1} \gamma_{i} f\left(x_{i}\right)\left[1-F\left(x_{i}\right)\right]^{m} \\
\times\left[1-F\left(x_{n}\right)\right]^{k-1} f\left(x_{n}\right),(2)
\end{array}
$$

on the cone $F^{-1}(0)<x_{1}<\ldots<x_{n}<F^{-1}(1)$ of $R^{n}$, where $\tilde{m}=\left(m_{1}, m_{2}, \ldots, m_{n-1}\right) \in R^{n-1}, \gamma_{r}=k+(n-r)+M_{r}>0$,

such that

$$
M_{r}=\sum_{j=r}^{n-1} m_{j}, \gamma_{n}=k>0 \text {. }
$$

\section{Particular cases from (2):}

1- Ordinary order statistics: for $k=1$ and $\tilde{m}=0$. 
2- Type II right censored order statistics: for $k=1$ and $m_{i}=0, i=1,2, \ldots, n-1$, $m_{n}=n-r$.

3- Type II progressive censored order statistics: for $m_{i} \neq 0, i=1,2, \ldots, n-1$, $m_{n}=k-1$.

4- Record values for $k=1$ and $\tilde{m}=-1$.

\section{Main Results}

\subsection{Bayesian approach}

\subsubsection{Informative Prior on $\alpha$ and non-Informative Prior on $\beta$}

Suppose that $X(1, n, \tilde{m}, k), \ldots, X(n, n, \tilde{m}, k)$, are GOS from the generalized gamma distribution (1), thus the likelihood function is given by:

$$
\begin{aligned}
& L(\alpha, \beta ; X)=C \prod_{i=1}^{n} \frac{\alpha}{\beta}\left(x_{i} / \beta\right)^{\alpha \lambda-1} \exp \left[-\left(x_{i} / \beta\right)^{\alpha}\right] \\
& \times \prod_{i=1}^{n-1}\left[\Gamma(\lambda)-I G\left(\lambda,\left(x_{i} / \beta\right)^{\alpha}\right)\right]^{m} i\left[\Gamma(\lambda)-I G\left(\lambda,\left(x_{n} / \beta\right)^{\alpha}\right)\right]^{k-1}
\end{aligned}
$$

Under the assumption that the shape parameter $\alpha$ has a conjugate gamma prior, it is assumed that the prior distribution of $\alpha$ has Gamma (a, b), with PDF

$$
\Pi_{1}(\alpha \mid a, b)=\frac{b^{-a}}{\Gamma(a)} \alpha^{a-1} e^{-\alpha / b}, \alpha>0,
$$

where, the hyper-parameters $a>0, b>0$. 
Bayesian Inference on the Generalized Gamma Distribution

By choosing the non-informative prior density for $\beta$ which is defined as:

$$
\Pi_{2}(\beta) \propto 1 / \beta,(\beta>0) .
$$

Thus, the joint prior density for $\alpha$ and $\beta$ has the form:

$\Pi(\alpha, \beta \mid a, b) \propto \frac{b^{-a}}{\Gamma(a) \beta} \alpha^{a-1} e^{-\alpha / b}, \quad a>0, b>0$.

Thus, using Bayes' theorem, the joint posterior PDF of $\alpha$ and $\beta$ can be written as:

$$
g(\alpha, \beta \mid \mathrm{x}) \propto \Pi(\alpha, \beta \mid a, b) \mathrm{L}(\alpha, \beta ; \mathrm{x}) .
$$

Substituting (3) and (4) in (5), we get

$$
\begin{aligned}
& g(\alpha, \beta \mid \mathrm{x} ; \lambda)=\mathrm{C} \alpha^{n+a-1} \beta^{-n \lambda \alpha-1} \prod_{i=1}^{n} x_{i}^{\alpha \lambda} \\
& \times \exp \left[\sum_{i=1}^{n} m_{i} \ln \left[\Gamma(\lambda)-I G\left(\lambda,\left(x_{i} / \beta\right)^{\alpha}\right)\right]-\sum_{i=1}^{n}\left(x_{i} / \beta\right)^{\alpha}-\alpha / b\right] \\
& , \quad m_{n}=k-1 .
\end{aligned}
$$

$C$ is the normalizing constant and is given by:

$$
\begin{aligned}
& \mathrm{C}^{-1}=\int_{0}^{\infty} \int_{0}^{\infty} \alpha^{n+a-1} \beta^{-n \lambda \alpha-1} \prod_{i=1}^{n} x_{i}^{\alpha \lambda} \\
& \times \exp \left[\sum_{i=1}^{n} m_{i} \ln \left[\Gamma(\lambda)-I G\left(\lambda,\left(x_{i} / \beta\right)^{\alpha}\right)\right]-\sum_{i=1}^{n}\left(x_{i} / \beta\right)^{\alpha}-\alpha / b\right] d \beta d \alpha .
\end{aligned}
$$

The marginal densities for $\alpha$ (or $\beta$ ) can be derived by integrating with respect to $\beta$ (or $\alpha$ ) out of (6) respectively as: 


$$
\begin{aligned}
& g_{1}(\alpha \mid \text { data })=C \int_{0}^{\infty} \alpha^{n+a-1} \beta^{-n \lambda \alpha-1} \prod_{i=1}^{n} x_{i}^{\alpha \lambda} \\
& \times \exp \left[\sum_{i=1}^{n} m_{i} \ln \left[\Gamma(\lambda)-I G\left(\lambda,\left(x_{i} / \beta\right)^{\alpha}\right)\right]-\sum_{i=1}^{n}\left(x_{i} / \beta\right)^{\alpha}-\alpha / b\right] d \beta .
\end{aligned}
$$

Similarly

$$
\begin{aligned}
& g_{2}(\beta \mid \text { data })=C \int_{0}^{\infty} \alpha^{n+a-1} \beta^{-n \lambda \alpha-1} \prod_{i=1}^{n} x_{i}^{\alpha \lambda} \\
& \times \exp \left[\sum_{i=1}^{n} m_{i} \ln \left[\Gamma(\lambda)-I G\left(\lambda,\left(x_{i} / \beta\right)^{\alpha}\right)\right]-\sum_{i=1}^{n}\left(x_{i} / \beta\right)^{\alpha}-\alpha / b\right] d \alpha .
\end{aligned}
$$

\subsubsection{Non-informative Prior on $\alpha$ and $\beta$}

When the parameters $\alpha$ and $\beta$ are unknown, a conjugate family of a continuous joint prior density on $\alpha$ and $\beta$ does not exist. In trying to solve this problem and simplify the Bayesian analysis, the arguments in Box and Tiao (1973) have been used, for deriving the non-informative joint prior density for $\alpha$ and $\beta$, when $\lambda$ is known as:

$$
\Pi(\alpha, \beta, \lambda) \propto 1 / \alpha \beta .
$$

Thus using (3) and (9) in (5), the joint posterior density of $\alpha$ and $\beta$ can be written as:-

$$
g^{*}(\alpha, \beta \mid \mathrm{x} ; \lambda)=\mathrm{C}_{1} \alpha^{n-1} \beta^{-n \alpha \lambda-1} \prod_{i=1}^{n} x_{i}^{\alpha \lambda}
$$


Bayesian Inference on the Generalized Gamma Distribution

$$
\times \exp \left[\sum_{i=1}^{n} m_{i} \ln \left[\Gamma(\lambda)-I G\left(\lambda,\left(x_{i} / \beta\right)^{\alpha}\right)\right]-\sum_{i=1}^{n}\left(x_{i} / \beta\right)^{\alpha}\right],
$$

$m_{n}=k-1$.

$C_{1}$ is the normalizing constant and is given by:

$$
\begin{aligned}
& \mathrm{C}_{1}^{-1}=\int_{0}^{\infty} \int_{0}^{\infty} \alpha^{n-1} \beta^{-n \alpha \lambda-1} \prod_{i=1}^{n} x_{i}^{\alpha \lambda} \\
& \times \exp \left[\sum_{i=1}^{n} m_{i} \ln \left[\Gamma(\lambda)-I G\left(\lambda,\left(x_{i} / \beta\right)^{\alpha}\right)\right]-\sum_{i=1}^{n}\left(x_{i} / \beta\right)^{\alpha}\right] d \beta d \alpha .
\end{aligned}
$$

The marginal densities of $\alpha$ and $\beta$ are obtained respectively as:

$$
\begin{aligned}
& g_{1}^{*}(\alpha \mid \text { data })=C_{1} \int_{0}^{\infty} \alpha^{n-1} \beta^{-n \alpha \lambda-1} \prod_{i=1}^{n} x_{i}^{\alpha \lambda} \\
& \times \exp \left[\sum_{i=1}^{n} m_{i} \ln \left[\Gamma(\lambda)-I G\left(\lambda,\left(x_{i} / \beta\right)^{\alpha}\right)\right]-\sum_{i=1}^{n}\left(x_{i} / \beta\right)^{\alpha}\right] d \beta .
\end{aligned}
$$

Similarly

$$
\begin{aligned}
& g_{2}^{*}(\beta \mid \text { data })=C_{1} \int_{0}^{\infty} \alpha^{n-1} \beta^{-n \alpha \lambda-1} \prod_{i=1}^{n} x_{i}^{\alpha \lambda} \\
& \quad \times \exp \left[\sum_{i=1}^{n} m_{i} \ln \left[\Gamma(\lambda)-I G\left(\lambda,\left(x_{i} / \beta\right)^{\alpha}\right)\right]-\sum_{i=1}^{n}\left(x_{i} / \beta\right)^{\alpha}\right] d \alpha .
\end{aligned}
$$

\subsection{Asymptotic maximum likelihood estimation}

The MLE is a popular statistical method used for deriving the classical confidence intervals for the distribution parameters. It provides statistical studies for the 
parameters and can be regarded as reference technique as in our study. For purpose of comparison, we obtain the confidence intervals for the parameters, thus the asymptotic variance- covariance matrix of the MLEs can be derived, which is the inversion of the Fisher information matrix whose elements are the negative of the expected values of the second order partial derivatives of the logarithm of the likelihood function. The log likelihood function based on the first $\mathrm{n}$ GOS from the GGD (1), can be derived as:

$$
\begin{aligned}
\ln L(\alpha, \beta, \lambda)= & C+n \ln (\alpha)-n \alpha \lambda \ln (\beta)+(\alpha \lambda-1) \sum_{i=1}^{n} \ln \left(x_{i}\right)-\sum_{i=1}^{n}\left(x_{i} / \beta\right)^{\alpha} \\
& +\sum_{i=1}^{n} m_{i} \ln \left[\Gamma(\lambda)-I G\left(\lambda,\left(x_{i} / \beta\right)^{\alpha}\right)\right] \\
\text { where } m_{n} & =k-1 .
\end{aligned}
$$

The maximum likelihood estimators $\hat{\alpha}$ and $\hat{\beta}$ are the solutions to the system of equations obtained by equating to zero the first partial derivatives of the natural logarithm of the likelihood function with respect to $\alpha$ and $\beta$ when $\lambda$ is known.

Thus, the ML estimators $\hat{\alpha}$ and $\hat{\beta}$ for $\alpha$ and $\beta$, respectively, can be obtained from the solution of the following normal equations:

$$
\begin{gathered}
\frac{\partial \ln L}{\alpha}=\frac{n}{\alpha}-n \lambda \ln \beta+\lambda \sum_{i=1}^{n} \ln x_{i}-\sum_{i=1}^{n}\left(x_{i} / \beta\right)^{\alpha} \ln \left(x_{i} / \beta\right) \\
-\sum \frac{*\left(x_{i} / \beta\right)^{\alpha \lambda} \exp \left[-\left(x_{i} / \beta\right)^{\alpha}\right] \ln \left(x_{i} / \beta\right)}{\Gamma(\lambda)-I G\left(\lambda,\left(x_{i} / \beta\right)^{\alpha}\right)}=0,
\end{gathered}
$$

in expression (15) and later expressions, we use for convenience the summation notation 
Bayesian Inference on the Generalized Gamma Distribution

$$
\begin{aligned}
& \sum^{*} g_{i}=\sum_{i=1}^{n} m_{i} g_{i}, \quad m_{n}=k-1 . \\
& \frac{\partial \ln L}{\beta}=-\frac{n \alpha \lambda}{\beta}+\frac{\alpha}{\beta} \sum_{i=1}^{n}\left(x_{i} / \beta\right)^{\alpha}+\frac{\alpha}{\beta} \sum^{*} \frac{\left(x_{i} / \beta\right)^{\alpha \lambda} \exp \left[-\left(x_{i} / \beta\right)^{\alpha}\right]}{\Gamma(\lambda)-I G\left(\lambda,\left(x_{i} / \beta\right)^{\alpha}\right)}=0 .
\end{aligned}
$$

Equations (15) and (16) can't be solved analytically. Numerical technique such as the Newton Raphson method can be used to solve these equations numerically. Thus, the Fisher information matrix $\boldsymbol{I}(\boldsymbol{\theta})$ can be constructed by differentiating (14) with respect to $\alpha$ and $\beta$ respectively when $\hat{\alpha}$ and $\hat{\beta}$ are known.

$$
\begin{aligned}
& I_{\alpha \alpha}=-\frac{\partial^{2} L n L}{\partial \alpha^{2}}=\frac{n}{\alpha^{2}}+\sum_{i=1}^{n}\left(x_{i} / \beta\right)^{\alpha}\left(\ln \left(x_{i} / \beta\right)\right)^{2}+\sum^{*}\left[\frac{\left(x_{i} / \beta\right)^{\alpha \lambda} \exp \left[\left(x_{i} / \beta\right)^{\alpha}\right] \ln \left(x_{i} / \beta\right)}{\Gamma(\lambda)-I G\left(\lambda,\left(x_{i} / \beta\right)^{\alpha}\right)}\right]^{2} \\
&+\sum^{*} \frac{\left.\left(x_{i} / \beta\right)^{\alpha \lambda} \exp \left[-\left(x_{i} / \beta\right)^{\alpha}\right] \ln \left(x_{i} / \beta\right)\right)^{2}\left(\lambda-\left(x_{i} / \beta\right)^{\alpha}\right)}{\Gamma(\lambda)-I G\left(\lambda,\left(x_{i} / \beta\right)^{\alpha}\right)}, \\
& I_{\beta \beta}=-\frac{\partial^{2} \log L}{\partial \beta^{2}}=-\frac{n \alpha \lambda}{\beta^{2}}+\frac{\alpha(\alpha+1)}{\beta^{2}} \sum_{i=1}^{n}\left(x_{i} / \beta\right)^{\alpha}+\frac{\alpha}{\beta^{2}} \sum^{*}\left[\frac{\left(x_{i} / \beta\right)^{\alpha \lambda} \exp \left[-\left(x_{i} / \beta\right)^{\alpha}\right]}{\Gamma(\lambda)-\operatorname{Ig}\left(\lambda,\left(x_{i} / \beta\right)^{\alpha}\right)}\right] \\
&-\frac{\alpha^{2}}{\beta^{2}} \sum^{*}\left(\frac{\left(x_{i} / \beta\right)^{\alpha \lambda} \exp \left[-\left(x_{i} / \beta\right)^{\alpha}\right]\left[-\lambda+\left(x_{i} / \beta\right)^{\alpha}\right]}{\Gamma(\lambda)-I G\left(\lambda,\left(x_{i} / \beta\right)^{\alpha}\right)}-\left[\frac{\left(x_{i} / \beta\right)^{\alpha \lambda} \exp \left[-\left(x_{i} / \beta\right)^{\alpha}\right]}{\Gamma(\lambda)-I G\left(\lambda,\left(x_{i} / \beta\right)^{\alpha}\right)}\right]\right.
\end{aligned}
$$

and

$$
I_{\alpha \beta}=-\frac{\partial^{2} \operatorname{LnL}}{\partial \alpha \partial \beta}=\frac{n \lambda}{\beta}-\frac{\alpha}{\beta} \sum_{i=1}^{n}\left(x_{i} / \beta\right)^{\alpha} \ln \left(x_{i} / \beta\right)-\frac{1}{\beta} \sum_{i=1}^{n}\left(x_{i} / \beta\right)^{\alpha}
$$




$$
\begin{gathered}
-\Sigma * \frac{\frac{1}{\beta}\left(x_{i} / \beta\right)^{\alpha \lambda} \exp \left[-\left(x_{i} / \beta\right)^{\alpha}\right]\left[1-\alpha\left(x_{i} / \beta\right)^{\alpha} \ln \left(x_{i} / \beta\right)+\alpha \lambda \ln \left(x_{i} / \beta\right)\right]}{\Gamma(\lambda)-I G\left(\lambda,\left(x_{i} / \beta\right)^{\alpha}\right)} \\
-\Sigma * \frac{\frac{\alpha}{\beta}\left[\left(x_{i} / \beta\right)^{\alpha \lambda} \exp \left(-\left(x_{i} / \beta\right)^{\alpha}\right)\right]^{2} \log \left(x_{i} / \beta\right)}{\left[\Gamma(\lambda)-I G\left(\lambda,\left(x_{i} / \beta\right)^{\alpha}\right)\right]^{2}}
\end{gathered}
$$

Thus, the elements of $\boldsymbol{I}(\boldsymbol{\theta})$ have been evaluated from (17), (18) and (19), by substituting $\hat{\alpha}$ and $\hat{\beta}$ instead of $\alpha$ and $\beta$. Therefore, the asymptotic Fisher's information matrix can be written as

$$
F=\left[\begin{array}{cc}
I_{\alpha \alpha} & I_{\alpha \beta} \\
I_{\beta \alpha} & I_{\beta \beta}
\end{array}\right]_{(\alpha, \beta)=(\hat{\alpha}, \hat{\beta})}
$$

In relation to the asymptotic variance-covariance matrix of the ML estimators of the parameters, it can be approximated numerically by inverting the above Fisher's information matrix F. The approximate $100(1-\gamma) \%$ two-sided confidence intervals for $\alpha$ and $\beta$ can be, respectively, obtained as

$$
\hat{\alpha} \pm Z_{\gamma / 2} \hat{\sigma}(\hat{\alpha}) \text { and } \hat{\beta} \pm Z_{\gamma / 2} \hat{\sigma}(\hat{\beta}),
$$

where $Z_{\gamma / 2}$ is the upper $(\gamma / 2)^{\text {th }}$ percentile of a standard normal distribution, and $\hat{\sigma}(\hat{\alpha}), \hat{\sigma}(\hat{\beta})$ are, respectively, the standard deviations of the ML estimators of the parameters $\alpha$ and $\beta$.

\section{Simulation Study and Comparisons}

To assess the performance of the confidence intervals based on the Bayesian approach comparing to those based on the asymptotic maximum likelihood 
estimation approach, Monte Carlo simulations, are carried out in terms of the following criteria:

i) Covering percentage (CP), which is defined as the fraction of times the confidence interval covers the true value of the parameter in repeated sampling.

ii) The mean length of intervals (MLI).

iii) The standard error of the covering percentage (SDE), which is defined for the nominal level $(1-\alpha) 100 \%$ by $S D E(\hat{\alpha})=\sqrt{\frac{\hat{\alpha}(1-\hat{\alpha})}{M}}$, where $(1-\hat{\alpha}) 100 \%$ denote the corresponding Monte Carlo estimate and $\mathrm{M}$ is the number of Monte Carlo trials. Thus, for the nominal level 95\% and 1000 simulation trails, say, the standard error of the covering percentage is 0.0049 , which is approximately $\pm 1 \%$. Therefore, we say the procedure is adequate if the SDE is within $\pm 2 \%$ error for the nominal level 95\%.

The results, based on 1000 Monte Carlo simulations are given for samples of sizes $\mathrm{n}$ $=20$ and 40, which have been generated for the scale parameter $\beta=2$, 3, shape parameter $\alpha=1,2,3$ and

$\lambda=1,2$, based on the complete, the type-II censored and the type-II progressive censored samples with binomial random removals at $\mathrm{P}=0.5$ with uncensored levels $\mathrm{r}$ equal to [n/2] and [3n/4]. The corresponding hyper-parameters provided in the previous section are taking to be $a=2.0$ and $b=2.0$. From the simulation results, we summarized some of the interesting features in the following points:

1. The results in Tables (1-4) indicated that, as the sample size increases, the two approaches have values of $M L I \mathrm{~s}$ decrease and the values of $C P \mathrm{~s}$ increase, while the values of $S D E$ s decrease for all values of $\alpha$ based on the complete and censored samples.

2. The mean length of intervals for the parameter $\alpha$ increase as the shape parameter increases as would be expected. On the contrary, for the parameter $\beta$ the mean length of the intervals decrease as the shape parameter $\alpha$ increases based on the complete, type-II censored and type-II progressive censored samples.

3. As the true value $\lambda$ increases the MLIs decrease, and the CPs mostly increase and the $S D E s$ decrease based on the complete, type-II censored 
and type-II progressive censored samples for the parameter $\alpha$ and $\beta$, for both approaches.

4. As the uncensored level increases the length of intervals decrease for the parameter $\alpha$ and $\beta$ for both approaches.

5. For the parameter $\alpha$, as the true value of $\alpha$ increases, the values of $C P s$ and $S D E s$ are fixed, based on the Classical approach.

6. The Bayesian approach is conservative for estimating the parameters $\alpha$ and $\beta$ because the covering percentages are much greater than the nominal level than those based on the Classical inference for all sample sizes. On the contrary, the classical approach is anti-conservative for estimating $\alpha$ and almost conservative for estimating $\beta$ when the sample size is greater than 20 .

7. It is worthwhile to note that, the values of MLIs and SDEs based on the type-II progressive censored samples are less than those based on typeII censored samples. Moreover, the CPs for type-II progressive censored samples are greater than those based on type-II censored samples.

8. Both the two approaches are adequate because the values of $S D E$ s are less than \pm 2 for the nominal level $95 \%$.

9. Finally, in general, we can conclude that the length of intervals for $\alpha$ and $\beta$ based on the informative prior are less than those based on the non-informative prior, and the CPs based on the informative prior are greater than those based on the non- informative prior as expected.

\section{An illustrative Example}

Consider the results of tests, the endurance of deep groove ball bearings. The data are quoted from Lawless (1982) consist of a complete sample of size $n=23$, that represent the results of the test, in millions of revolutions before failures are:

17.88, 28.92, 33.00, 41.52, 41.12, 45.60, 48.48, 51.84, 51.96, 54.12, 55.56, 67.80, 68.64, 68.64, 68.88, 84.12, 93.12, 98.64, 105.12, 105.84, 127.92, 128.04, 173.40. 
Thus, for the purpose of comparison, we derived the confidence intervals of the unknown parameters $\alpha$ and $\beta$ based on these data for complete, type-II censored and type-II progressively censored samples with binomial removals at $\mathrm{P}=0.5$ and uncensored levels are equal to [n/2] and [3n/4] at $\lambda=1$, 2, we set the hyperparameters for the informative prior as $a=2.0$ and $b=2.0$. From the result in Tables 5, we conclude the following points:

1. The length of intervals based on the informative prior, and the non-informative are smaller than those based on the classical approach for the parameter $\alpha$ and $\beta$, based on complete, type-II censored and type-II progressive censored samples.

2. As the uncensored level increases the length of intervals decrease for the parameter $\alpha$ and $\beta$ for both approaches.

3. As the true value $\lambda$ increases, the length of intervals for the parameter $\alpha$ and $\beta$ decrease based on the two approaches.

4. It is worthwhile to note that, the length of intervals for $\alpha$ and $\beta$ based on the type-II progressive censored samples are less than those based on type-II censored sample based on the two approaches.

5. The length of intervals based on the informative prior are less than those based on the non- informative prior as expected.

We conclude that the results based on the real data ensure the simulation results.

\section{Conclusions}

In this paper, the confidence intervals for the unknown parameters of the GGD have been constructed based on the Bayesian approach using the informative and the noninformative priors, and the Asymptotic maximum likelihood method based on the complete, the type-II censored and the type-II progressive censored samples. We compared the performance of the two approaches, via Monte Carlo simulations and some real data. From the simulation results, it is observed that the Bayesian confidence intervals based on the informative, and the non-informative priors outperform the confidence intervals based on the Asymptotic maximum likelihood method. 
Table 1. The (MLIs), (CPs) and (SDEs) for the Bayesian and the classical approaches when the nominal level is $95 \%$ for the parameter $\boldsymbol{\alpha}$ with $(\boldsymbol{\beta}=2,3$ ) based on the type-II progressive censored samples with uncensored levels (50\% and 75\%).

\begin{tabular}{|c|c|c|c|c|c|c|c|c|c|c|c|c|}
\hline \multicolumn{4}{|c|}{ Approaches } & \multicolumn{3}{|c|}{ Inform Prior } & \multicolumn{3}{|c|}{ Non Inform Prior } & \multicolumn{3}{|c|}{ Classical } \\
\hline$n$ & $r$ & $\lambda$ & $\alpha$ & MLI & $\mathrm{CP}$ & SE & MLI & $\mathrm{CP}$ & SDE & MLI & $\mathrm{CP}$ & SDE \\
\hline \multirow[t]{12}{*}{20} & \multirow[t]{3}{*}{10} & \multirow[t]{3}{*}{1} & 1 & 1.1025 & 0.925 & 0.0083 & 1.1371 & 0.941 & 0.0075 & 1.1495 & \multirow[t]{3}{*}{0.940} & \multirow[t]{3}{*}{0.0075} \\
\hline & & & 2 & 2.0042 & 0.951 & 0.0068 & 2.0519 & 0.960 & 0.0062 & 2.2990 & & \\
\hline & & & 3 & 2.1304 & 0.983 & 0.0041 & 2.2371 & 0.974 & 0.0050 & 3.4485 & & \\
\hline & \multirow[t]{3}{*}{15} & & 1 & 0.9024 & 0.935 & 0.0078 & 0.8955 & 0.963 & 0.0060 & 0.8865 & \multirow[t]{3}{*}{0.959} & \multirow[t]{3}{*}{0.0063} \\
\hline & & & 2 & 1.7065 & 0.957 & 0.0064 & 1.7298 & 0.967 & 0.0056 & 1.7725 & & \\
\hline & & & 3 & 1.8920 & 0.988 & 0.0034 & 1.9559 & 0.984 & 0.0040 & 2.6594 & & \\
\hline & \multirow[t]{3}{*}{10} & 2 & 1 & 0.9703 & 0.952 & 0.0068 & 0.9825 & 0.969 & 0.0055 & 1.1073 & \multirow[t]{3}{*}{0.951} & \multirow[t]{3}{*}{0.0068} \\
\hline & & & 2 & 1.5764 & 0.994 & 0.0024 & 1.6585 & 0.989 & 0.0033 & 2.2145 & & \\
\hline & & & 3 & 2.0937 & 0.993 & 0.0026 & 2.2004 & 0.989 & 0.0033 & 3.3218 & & \\
\hline & \multirow[t]{3}{*}{15} & & 1 & 0.7934 & 0.948 & 0.0070 & 0.8541 & 0.961 & 0.0061 & 0.8482 & \multirow[t]{3}{*}{0.952} & \multirow[t]{3}{*}{0.0068} \\
\hline & & & 2 & 1.3995 & 0.979 & 0.0045 & 1.4440 & 0.981 & 0.0043 & 1.6965 & & \\
\hline & & & 3 & 1.8571 & 0.992 & 0.0028 & 1.9188 & 0.990 & 0.0031 & 2.5447 & & \\
\hline \multirow[t]{12}{*}{40} & \multirow[t]{3}{*}{20} & \multirow[t]{3}{*}{1} & 1 & 0.7484 & 0.939 & 0.0076 & 0.7550 & 0.952 & 0.0068 & 0.7396 & \multirow[t]{3}{*}{0.949} & \multirow[t]{3}{*}{0.0070} \\
\hline & & & 2 & 1.3048 & 0.968 & 0.0056 & 1.4917 & 0.954 & 0.0066 & 1.4792 & & \\
\hline & & & 3 & 1.7406 & 0.990 & 0.0031 & 1.7835 & 0.983 & 0.0041 & 2.2188 & & \\
\hline & \multirow[t]{3}{*}{30} & & 1 & 0.6138 & 0.932 & 0.0099 & 0.6114 & 0.945 & 0.0072 & 0.5915 & \multirow[t]{3}{*}{0.944} & \multirow[t]{3}{*}{0.0073} \\
\hline & & & 2 & 1.2142 & 0.940 & 0.0075 & 1.2200 & 0.945 & 0.0072 & 1.1831 & & \\
\hline & & & 3 & 1.5320 & 0.974 & 0.0050 & 1.5562 & 0.976 & 0.0048 & 1.7746 & & \\
\hline & \multirow[t]{3}{*}{20} & 2 & 1 & 0.6871 & 0.940 & 0.0075 & 0.6906 & 0.957 & 0.0064 & 0.7087 & \multirow[t]{3}{*}{0.939} & \multirow[t]{3}{*}{0.0076} \\
\hline & & & 2 & 1.2650 & 0.963 & 0.0060 & 1.2920 & 0.966 & 0.0057 & 1.4174 & & \\
\hline & & & 3 & 1.6912 & 0.981 & 0.0043 & 1.7316 & 0.975 & 0.0049 & 2.1261 & & \\
\hline & \multirow[t]{3}{*}{30} & & 1 & 0.5690 & 0.936 & 0.0077 & 0.5875 & 0.942 & 0.0074 & 0.5668 & 0.935 & 0.0078 \\
\hline & & & 2 & 1.0957 & 0.948 & 0.0070 & 1.1083 & 0.946 & 0.0071 & 1.1336 & & \\
\hline & & & 3 & 1.4893 & 0.967 & 0.0056 & 1.5119 & 0.963 & 0.0060 & 1.7004 & & \\
\hline
\end{tabular}


Table 2. The (MLIs), (CPs) and (SDEs) for the Bayesian and the classical approaches when the nominal level is $95 \%$ for the parameter $\boldsymbol{\alpha}$ with $(\boldsymbol{\beta}=2,3$ ) based on the complete and type-II censored samples with uncensored levels ( $50 \%$ and $75 \%$ ).

\begin{tabular}{|c|c|c|c|c|c|c|c|c|c|c|c|c|c|}
\hline \multicolumn{5}{|c|}{ Approaches } & \multicolumn{3}{|c|}{ Inform Prior } & \multicolumn{3}{|c|}{ Non Inform Prior } & \multicolumn{3}{|c|}{ Classical } \\
\hline$n$ & $\lambda$ & $\beta$ & $r$ & $\alpha$ & MLI & CP & SDE & MLI & CP & SDE & MLI & CP & SDE \\
\hline \multirow[t]{18}{*}{20} & 1 & 2 & 10 & 1 & 1.3204 & 0.941 & 0.0075 & 1.3339 & 0.955 & 0.0066 & 1.3914 & 0.960 & 0.0062 \\
\hline & & & & 2 & 2.6137 & 0.950 & 0.0069 & 2.6722 & 0.954 & 0.0066 & 2.7828 & & \\
\hline & & & & 3 & 3.6015 & 0.970 & 0.0054 & 3.8193 & 0.957 & 0.0064 & 4.1742 & & \\
\hline & & & 15 & 1 & 1.0022 & 0.931 & 0.0080 & 0.9856 & 0.957 & 0.0064 & 0.9890 & 0.958 & 0.0063 \\
\hline & & & & 2 & 1.9285 & 0.951 & 0.0068 & 1.9495 & 0.956 & 0.0065 & 1.9780 & & \\
\hline & & & & 3 & 2.7010 & 0.960 & 0.0062 & 2.8010 & 0.957 & 0.0064 & 2.9669 & & \\
\hline & & & 20 & 1 & 0.7348 & 0.941 & 0.0075 & 0.7362 & 0.958 & 0.0063 & 0.7415 & 0.958 & 0.0063 \\
\hline & & & & 2 & 1.4499 & 0.951 & 0.0068 & 1.4672 & 0.956 & 0.0065 & 1.4831 & & \\
\hline & & & & 3 & 2.1457 & 0.961 & 0.0061 & 2.1983 & 0.956 & 0.0065 & 2.2246 & & \\
\hline & 2 & 2 & 10 & 1 & 1.2791 & 0.938 & 0.0076 & 1.2573 & 0.959 & 0.0063 & 1.2913 & 0.961 & 0.0061 \\
\hline & & & & 2 & 2.2393 & 0.962 & 0.0060 & 2.2878 & 0.961 & 0.0061 & 2.5826 & & \\
\hline & & & & 3 & 2.7468 & 0.974 & 0.0050 & 2.8652 & 0.966 & 0.0057 & 3.8738 & & \\
\hline & & & 15 & 1 & 0.9434 & 0.922 & 0.0085 & 0.9308 & 0.950 & 0.0069 & 0.9356 & 0.947 & 0.0071 \\
\hline & & & & 2 & 1.7218 & 0.948 & 0.0070 & 1.7507 & 0.951 & 0.0068 & 1.8712 & & \\
\hline & & & & 3 & 2.0187 & 0.979 & 0.0045 & 2.0909 & 0.975 & 0.0049 & 2.8067 & & \\
\hline & & & 20 & 1 & 0.7039 & 0.927 & 0.0082 & 0.7087 & 0.937 & 0.0077 & 0.7118 & 0.931 & 0.0080 \\
\hline & & & & 2 & 1.3926 & 0.935 & 0.0078 & 1.4121 & 0.938 & 0.0076 & 1.4236 & & \\
\hline & & & & 3 & 2.0661 & 0.939 & 0.0076 & 2.1155 & 0.939 & 0.0076 & 2.1354 & & \\
\hline \multirow[t]{16}{*}{40} & 1 & 2 & 20 & 1 & 0.8861 & 0.938 & 0.0076 & 0.8961 & 0.947 & 0.0071 & 0.8964 & 0.950 & 0.0069 \\
\hline & & & & 2 & 1.7832 & 0.938 & 0.0076 & 1.7961 & 0.948 & 0.0070 & 1.7928 & & \\
\hline & & & & 3 & 2.4983 & 0.948 & 0.0070 & 2.5747 & 0.949 & 0.0070 & 2.6892 & & \\
\hline & & & 30 & 1 & 0.6773 & 0.943 & 0.0073 & 0.6711 & 0.955 & 0.0066 & 0.6608 & 0.953 & 0.0067 \\
\hline & & & & 2 & 1.3359 & 0.951 & 0.0068 & 1.3404 & 0.956 & 0.0065 & 1.3217 & & \\
\hline & & & & 3 & 1.8515 & 0.956 & 0.0065 & 1.8781 & 0.953 & 0.0067 & 1.9825 & & \\
\hline & & & 40 & 1 & 0.5009 & 0.948 & 0.0070 & 0.5050 & 0.959 & 0.0063 & 0.5032 & 0.955 & 0.0066 \\
\hline & & & & 2 & 0.9957 & 0.953 & 0.0067 & 1.0043 & 0.959 & 0.0063 & 1.0063 & & \\
\hline & & & & 3 & 1.4843 & 0.958 & 0.0063 & 1.5037 & 0.959 & 0.0063 & 1.5095 & & \\
\hline & 2 & 2 & 20 & 1 & 0.8582 & 0.929 & 0.0081 & 0.8482 & 0.950 & 0.0069 & 0.8453 & 0.951 & 0.0068 \\
\hline & & & & 2 & 1.6467 & 0.941 & 0.0075 & 1.6619 & 0.950 & 0.0069 & 1.6905 & & \\
\hline & & & & 3 & 1.9835 & 0.966 & 0.0057 & 2.0389 & 0.967 & 0.0056 & 2.5358 & & \\
\hline & & & 30 & 1 & 0.6207 & 0.950 & 0.0069 & 0.6393 & 0.948 & 0.0070 & 0.6282 & 0.949 & 0.0070 \\
\hline & & & & 3 & 1.4837 & 0.961 & 0.0061 & 1.6128 & 0.954 & 0.0066 & 1.8845 & & \\
\hline & & & 40 & 1 & 0.4807 & 0.934 & 0.0079 & 0.4859 & 0.944 & 0.0073 & 0.4832 & 0.939 & 0.0076 \\
\hline & & & & 3 & 1.4275 & 0.943 & 0.0073 & 1.4462 & 0.944 & 0.0073 & 1.4497 & & \\
\hline
\end{tabular}


Table 3. The (MLIs), (CPs) and (SDEs) for the Bayesian and the classical approaches when the nominal level is $95 \%$ for the parameter $\boldsymbol{\beta}$ based on complete and type-II censored samples with uncensored levels ( $50 \%$ and $75 \%)$.

\begin{tabular}{|c|c|c|c|c|c|c|c|c|c|c|c|c|c|}
\hline \multicolumn{5}{|c|}{ Approaches } & \multicolumn{3}{|c|}{ Inform Prior } & \multicolumn{3}{|c|}{ Non Inform Prior } & \multicolumn{3}{|c|}{ Classical } \\
\hline$n$ & $\lambda$ & $r$ & $\beta$ & $\alpha$ & MLI & $\mathrm{CP}$ & SDE & MLI & $\mathrm{CP}$ & SDE & MLI & $\mathrm{CP}$ & SDE \\
\hline \multirow[t]{18}{*}{20} & 1 & 10 & 2 & 1 & 4.7710 & 0.959 & 0.0063 & 7.0842 & 0.966 & 0.0057 & 2.5376 & 0.797 & 0.0127 \\
\hline & & & & 2 & 2.0640 & 0.963 & 0.0060 & 2.4179 & 0.964 & 0.0059 & 1.2487 & 0.827 & 0.0120 \\
\hline & & & & 3 & 1.4995 & 0.962 & 0.0060 & 1.6988 & 0.960 & 0.0062 & 0.8336 & 0.833 & 0.0118 \\
\hline & & & 3 & 1 & 6.9905 & 0.959 & 0.0063 & 10.3907 & 0.966 & 0.0057 & 3.8064 & 0.797 & 0.0127 \\
\hline & & & & 2 & 3.0881 & 0.963 & 0.0060 & 3.6106 & 0.964 & 0.0059 & 1.8730 & 0.827 & 0.0120 \\
\hline & & & & 3 & 2.2467 & 0.962 & 0.0060 & 2.5433 & 0.960 & 0.0062 & 1.2505 & 0.833 & 0.0118 \\
\hline & & 15 & 2 & 1 & 2.5309 & 0.955 & 0.0066 & 2.8220 & 0.966 & 0.0057 & 1.8998 & 0.893 & 0.0098 \\
\hline & & & & 2 & 1.2033 & 0.960 & 0.0062 & 1.2884 & 0.967 & 0.0056 & 0.9473 & 0.908 & 0.0091 \\
\hline & & & & 3 & 0.9424 & 0.981 & 0.0043 & 0.9740 & 0.982 & 0.0042 & 0.6331 & 0.913 & 0.0089 \\
\hline & & & 3 & 1 & 3.7892 & 0.955 & 0.0066 & 4.2217 & 0.966 & 0.0057 & 2.8497 & 0.893 & 0.0098 \\
\hline & & & & 2 & 1.8042 & 0.960 & 0.0062 & 1.9316 & 0.967 & 0.0056 & 1.4209 & 0.908 & 0.0091 \\
\hline & & & & 3 & 1.4135 & 0.981 & 0.0043 & 1.4608 & 0.982 & 0.0042 & 0.9497 & 0.913 & 0.0089 \\
\hline & & 20 & 2 & 1 & 2.0274 & 0.951 & 0.0068 & 2.0951 & 0.958 & 0.0063 & 1.7691 & 0.925 & 0.0083 \\
\hline & & & & 2 & 0.9984 & 0.958 & 0.0063 & 1.0260 & 0.961 & 0.0061 & 0.8816 & 0.925 & 0.0083 \\
\hline & & & & 3 & 0.7458 & 0.984 & 0.0040 & 0.7563 & 0.984 & 0.0040 & 0.5888 & 0.925 & 0.0083 \\
\hline & & & 3 & 1 & 3.0395 & 0.951 & 0.0068 & 3.1407 & 0.958 & 0.0063 & 2.6537 & 0.925 & 0.0083 \\
\hline & & & & 2 & 1.4975 & 0.958 & 0.0063 & 1.5389 & 0.961 & 0.0061 & 1.3223 & 0.925 & 0.0083 \\
\hline & & & & 3 & 1.1186 & 0.984 & 0.0040 & 1.1343 & 0.984 & 0.0040 & 0.8831 & 0.925 & 0.0083 \\
\hline \multirow[t]{16}{*}{20} & 2 & 10 & 2 & 1 & 1.9554 & 0.960 & 0.0062 & 2.1447 & 0.975 & 0.0049 & 1.6267 & 0.894 & 0.0097 \\
\hline & & & & 3 & 0.7765 & 0.989 & 0.0033 & 0.8207 & 0.990 & 0.0031 & 0.5390 & 0.887 & 0.0100 \\
\hline & & & 3 & 1 & 2.9313 & 0.959 & 0.0063 & 3.2144 & 0.975 & 0.0049 & 2.4401 & 0.894 & 0.0097 \\
\hline & & & & 2 & 1.5602 & 0.979 & 0.0045 & 1.6222 & 0.979 & 0.0045 & 1.2120 & 0.891 & 0.0099 \\
\hline & & & & 3 & 1.1646 & 0.989 & 0.0033 & 1.2309 & 0.990 & 0.0031 & 0.8086 & 0.887 & 0.0100 \\
\hline & & 15 & 2 & 1 & 1.7544 & 0.955 & 0.0066 & 1.8397 & 0.947 & 0.0071 & 1.6751 & 0.918 & 0.0087 \\
\hline & & & & 3 & 0.6914 & 0.970 & 0.0054 & 0.7114 & 0.970 & 0.0054 & 0.5523 & 0.912 & 0.0090 \\
\hline & & & 3 & 1 & 2.6312 & 0.955 & 0.0066 & 2.7591 & 0.947 & 0.0071 & 2.5127 & 0.918 & 0.0087 \\
\hline & & & & 2 & 1.3578 & 0.958 & 0.0063 & 1.4250 & 0.974 & 0.0050 & 1.2434 & 0.914 & 0.0089 \\
\hline & & & & 3 & 0.9951 & 0.978 & 0.0046 & 1.0672 & 0.970 & 0.0054 & 0.8284 & 0.912 & 0.0090 \\
\hline & & 20 & 2 & 1 & 1.7163 & 0.949 & 0.007 & 1.7498 & 0.955 & 0.0066 & 1.6911 & 0.926 & 0.0083 \\
\hline & & & & 2 & 0.8764 & 0.955 & 0.0066 & 0.9020 & 0.956 & 0.0065 & 0.8340 & 0.923 & 0.0084 \\
\hline & & & & 3 & 0.5985 & 0.968 & 0.0056 & 0.6102 & 0.966 & 0.0057 & 0.5550 & 0.919 & 0.0086 \\
\hline & & & 3 & 1 & 2.5742 & 0.949 & 0.007 & 2.6245 & 0.955 & 0.0066 & 2.5367 & 0.926 & 0.0083 \\
\hline & & & & 2 & 1.3146 & 0.955 & 0.0066 & 1.3530 & 0.956 & 0.0065 & 1.2511 & 0.923 & 0.0084 \\
\hline & & & & 3 & 0.8978 & 0.968 & 0.0056 & 0.9153 & 0.966 & 0.0057 & 0.8325 & 0.919 & 0.0086 \\
\hline
\end{tabular}


Bayesian Inference on the Generalized Gamma Distribution

Table 3. ( Continued)

\begin{tabular}{|c|c|c|c|c|c|c|c|c|c|c|c|c|c|}
\hline \multicolumn{5}{|c|}{ Approaches } & \multicolumn{3}{|c|}{ Inform Prior } & \multicolumn{3}{|c|}{ Non Inform Prior } & \multicolumn{3}{|c|}{ Classical } \\
\hline$n$ & $\lambda$ & $r$ & $\beta$ & $\alpha$ & MLI & $\mathrm{CP}$ & SDE & MLI & $\mathrm{CP}$ & SDE & MLI & $\mathrm{CP}$ & SDE \\
\hline \multirow[t]{18}{*}{40} & 1 & 20 & 2 & 1 & 2.4513 & 0.958 & 0.0063 & 2.8335 & 0.964 & 0.0059 & 1.8354 & 0.876 & 0.0104 \\
\hline & & & & 2 & 1.1965 & 0.965 & 0.0058 & 1.2527 & 0.966 & 0.0057 & 0.9176 & 0.897 & 0.0096 \\
\hline & & & & 3 & 0.8861 & 0.960 & 0.0062 & 0.9207 & 0.957 & 0.0064 & 0.6137 & 0.902 & 0.0094 \\
\hline & & & 3 & 1 & 3.6684 & 0.958 & 0.0063 & 4.2364 & 0.963 & 0.0060 & 2.7531 & 0.876 & 0.0104 \\
\hline & & & & 2 & 1.7311 & 0.962 & 0.0060 & 1.8778 & 0.966 & 0.0057 & 1.3764 & 0.897 & 0.0096 \\
\hline & & & & 3 & 1.3291 & 0.960 & 0.0062 & 1.3809 & 0.957 & 0.0064 & 0.9206 & 0.902 & 0.0094 \\
\hline & & 30 & 2 & 1 & 1.5987 & 0.959 & 0.0063 & 1.6742 & 0.965 & 0.0058 & 1.3803 & 0.940 & 0.0075 \\
\hline & & & & 2 & 0.7960 & 0.965 & 0.0058 & 0.8418 & 0.975 & 0.0049 & 0.6909 & 0.944 & 0.0073 \\
\hline & & & & 3 & 0.6143 & 0.991 & 0.003 & 0.6203 & 0.992 & 0.0028 & 0.4616 & 0.945 & 0.0072 \\
\hline & & & 3 & 1 & 2.3970 & 0.959 & 0.0063 & 2.5100 & 0.965 & 0.0058 & 2.0704 & 0.940 & 0.0075 \\
\hline & & & & 2 & 1.1939 & 0.965 & 0.0058 & 1.2292 & 0.969 & 0.0055 & 1.0364 & 0.944 & 0.0073 \\
\hline & & & & 3 & 0.9219 & 0.991 & 0.003 & 0.9308 & 0.992 & 0.0028 & 0.6923 & 0.945 & 0.0072 \\
\hline & & 40 & 2 & 1 & 1.3737 & 0.947 & 0.0071 & 1.3930 & 0.952 & 0.0068 & 1.2692 & 0.954 & 0.0066 \\
\hline & & & & 2 & 0.6998 & 0.958 & 0.0063 & 0.7077 & 0.961 & 0.0061 & 0.6351 & 0.946 & 0.0071 \\
\hline & & & & 3 & 0.5480 & 0.993 & 0.0026 & 0.5493 & 0.993 & 0.0026 & 0.4241 & 0.946 & 0.0071 \\
\hline & & & 3 & 1 & 2.0603 & 0.947 & 0.0071 & 2.0888 & 0.951 & 0.0068 & 1.9038 & 0.954 & 0.0066 \\
\hline & & & & 2 & 1.0498 & 0.958 & 0.0063 & 1.0617 & 0.961 & 0.0061 & 0.9527 & 0.946 & 0.0071 \\
\hline & & & & 3 & 0.8229 & 0.993 & 0.0026 & 0.8249 & 0.993 & 0.0026 & 0.6362 & 0.946 & 0.0071 \\
\hline \multirow[t]{18}{*}{40} & 2 & 20 & 2 & 1 & 1.3107 & 0.948 & 0.0070 & 1.3690 & 0.957 & 0.0064 & 1.1938 & 0.914 & 0.0089 \\
\hline & & & & 2 & 0.6877 & 0.957 & 0.0064 & 0.7112 & 0.963 & 0.0060 & 0.5938 & 0.914 & 0.0089 \\
\hline & & & & 3 & 0.5647 & 0.991 & 0.0030 & 0.5699 & 0.990 & 0.0031 & 0.3957 & 0.916 & 0.0088 \\
\hline & & & 3 & 1 & 1.9658 & 0.948 & 0.0070 & 2.0533 & 0.957 & 0.0064 & 1.7907 & 0.914 & 0.0089 \\
\hline & & & & 2 & 1.0317 & 0.957 & 0.0064 & 1.0670 & 0.963 & 0.0060 & 0.8906 & 0.914 & 0.0089 \\
\hline & & & & 3 & 0.8483 & 0.991 & 0.0030 & 0.8560 & 0.990 & 0.0031 & 0.5936 & 0.916 & 0.0088 \\
\hline & & 30 & 2 & 1 & 1.2531 & 0.950 & 0.0069 & 1.2896 & 0.950 & 0.0069 & 1.2116 & 0.930 & 0.0081 \\
\hline & & & & 2 & 0.6387 & 0.974 & 0.0050 & 0.6532 & 0.977 & 0.0047 & 0.6012 & 0.926 & 0.0083 \\
\hline & & & & 3 & 0.4770 & 0.958 & 0.0063 & 0.4803 & 0.955 & 0.0066 & 0.4004 & 0.928 & 0.0082 \\
\hline & & & 3 & 1 & 1.8796 & 0.950 & 0.0069 & 1.9343 & 0.950 & 0.0069 & 1.8175 & 0.930 & 0.0081 \\
\hline & & & & 2 & 0.9615 & 0.958 & 0.0063 & 0.9796 & 0.977 & 0.0047 & 0.9018 & 0.926 & 0.0083 \\
\hline & & & & 3 & 0.6346 & 0.966 & 0.0057 & 0.6453 & 0.965 & 0.0058 & 0.6006 & 0.928 & 0.0082 \\
\hline & & 40 & 2 & 1 & 1.2471 & 0.950 & 0.0069 & 1.2578 & 0.955 & 0.0066 & 1.2114 & 0.941 & 0.0075 \\
\hline & & & & 2 & 0.6374 & 0.957 & 0.0064 & 0.6463 & 0.962 & 0.0060 & 0.6004 & 0.937 & 0.0077 \\
\hline & & & & 3 & 0.3909 & 0.953 & 0.0067 & 0.4286 & 0.977 & 0.0047 & 0.3997 & 0.938 & 0.0076 \\
\hline & & & 3 & 1 & 1.8706 & 0.950 & 0.0069 & 1.8867 & 0.955 & 0.0066 & 1.8171 & 0.941 & 0.0075 \\
\hline & & & & 2 & 0.9562 & 0.957 & 0.0064 & 0.9694 & 0.962 & 0.0060 & 0.9007 & 0.937 & 0.0077 \\
\hline & & & & 3 & 0.5865 & 0.953 & 0.0067 & 0.5934 & 0.953 & 0.0067 & 0.5996 & 0.938 & 0.0076 \\
\hline
\end{tabular}


Table 4. The (MLIs), (CPs) and (SDEs) for the Bayesian and the classical approaches when the nominal level is $95 \%$ for the parameter $\boldsymbol{\beta}$ based on type-II progressive censored samples with uncensored levels ( $50 \%$ and $75 \%)$.

\begin{tabular}{|c|c|c|c|c|c|c|c|c|c|c|c|c|c|}
\hline \multicolumn{5}{|c|}{ Approaches } & \multicolumn{3}{|c|}{ Inform Prior } & \multicolumn{3}{|c|}{ Non Inform Prior } & \multicolumn{3}{|c|}{ Classical } \\
\hline$n$ & $\lambda$ & $r$ & $\beta$ & $\alpha$ & $M L I$ & $C P$ & $S D E$ & $M L I$ & $C P$ & $S D E$ & $M L I$ & $C P$ & $S D E$ \\
\hline \multirow[t]{24}{*}{20} & 1 & 10 & 2 & 1 & 2.3004 & 0.940 & 0.0075 & 2.4357 & 0.955 & 0.0066 & 2.3476 & 0.883 & 0.0102 \\
\hline & & & & 2 & 1.4266 & 0.947 & 0.0071 & 1.5197 & 0.953 & 0.0067 & 1.1793 & 0.896 & 0.0097 \\
\hline & & & & 3 & 1.0439 & 0.969 & 0.0055 & 1.0924 & 0.975 & 0.0049 & 0.7919 & 0.903 & 0.0094 \\
\hline & & & 3 & 1 & 2.3459 & 0.971 & 0.0053 & 2.4998 & 0.974 & 0.0050 & 3.5214 & 0.883 & 0.0102 \\
\hline & & & & 2 & 1.6806 & 0.958 & 0.0063 & 1.7713 & 0.966 & 0.0057 & 1.7689 & 0.896 & 0.0097 \\
\hline & & & & 3 & 1.3951 & 0.975 & 0.0049 & 1.4451 & 0.978 & 0.0046 & 1.1878 & 0.903 & 0.0094 \\
\hline & & 15 & 2 & 1 & 2.0478 & 0.933 & 0.0079 & 2.1302 & 0.943 & 0.0073 & 1.9795 & 0.893 & 0.0098 \\
\hline & & & & 2 & 1.1405 & 0.939 & 0.0076 & 1.1865 & 0.944 & 0.0073 & 0.9894 & 0.904 & 0.0093 \\
\hline & & & & 3 & 0.8005 & 0.960 & 0.0062 & 0.8205 & 0.963 & 0.0060 & 0.6622 & 0.908 & 0.0091 \\
\hline & & & 3 & 1 & 2.1108 & 0.969 & 0.0055 & 2.2078 & 0.970 & 0.0054 & 2.9692 & 0.893 & 0.0098 \\
\hline & & & & 2 & 1.4686 & 0.946 & 0.0071 & 1.5199 & 0.954 & 0.0066 & 1.4842 & 0.904 & 0.0093 \\
\hline & & & & 3 & 1.1544 & 0.962 & 0.0060 & 1.1799 & 0.964 & 0.0059 & 0.9932 & 0.908 & 0.0091 \\
\hline & 2 & 10 & 2 & 1 & 1.8015 & 0.980 & 0.0044 & 2.0945 & 0.970 & 0.0054 & 2.3464 & 0.903 & 0.0094 \\
\hline & & & & 2 & 1.1862 & 0.970 & 0.0054 & 1.2898 & 0.963 & 0.0060 & 1.1332 & 0.892 & 0.0098 \\
\hline & & & & 3 & 0.8386 & 0.952 & 0.0068 & 0.9037 & 0.971 & 0.0053 & 0.7506 & 0.892 & 0.0098 \\
\hline & & & 3 & 1 & 1.9329 & 0.979 & 0.0045 & 2.1408 & 0.969 & 0.0055 & 3.5196 & 0.903 & 0.0094 \\
\hline & & & & 2 & 1.2866 & 0.987 & 0.0036 & 1.4227 & 0.980 & 0.0044 & 1.6997 & 0.892 & 0.0098 \\
\hline & & & & 3 & 1.0568 & 0.977 & 0.0047 & 1.1326 & 0.972 & 0.0052 & 1.1259 & 0.892 & 0.0098 \\
\hline & & 15 & 2 & 1 & 1.6243 & 0.972 & 0.0052 & 1.8391 & 0.965 & 0.0058 & 1.9515 & 0.933 & 0.0079 \\
\hline & & & & 2 & 0.9957 & 0.965 & 0.0058 & 1.0480 & 0.963 & 0.0060 & 0.9511 & 0.927 & 0.0082 \\
\hline & & & & 3 & 0.6931 & 0.977 & 0.0047 & 0.7128 & 0.984 & 0.0040 & 0.6307 & 0.928 & 0.0082 \\
\hline & & & 3 & 1 & 1.6772 & 0.988 & 0.0034 & 1.8810 & 0.980 & 0.0044 & 2.9273 & 0.933 & 0.0079 \\
\hline & & & & 2 & 0.9086 & 0.960 & 0.0062 & 1.1647 & 0.990 & 0.0031 & 1.4266 & 0.927 & 0.0082 \\
\hline & & & & 3 & 0.8824 & 0.988 & 0.0034 & 0.9189 & 0.987 & 0.0036 & 0.9461 & 0.928 & 0.0082 \\
\hline
\end{tabular}


Bayesian Inference on the Generalized Gamma Distribution

Table 4. (continued)

\begin{tabular}{|c|c|c|c|c|c|c|c|c|c|c|c|c|c|}
\hline \multicolumn{5}{|c|}{ Approaches } & \multicolumn{3}{|c|}{ Inform Prior } & \multicolumn{3}{|c|}{ Non Inform Prior } & \multicolumn{3}{|c|}{ Classical } \\
\hline$n$ & $\lambda$ & $r$ & $\beta$ & $\alpha$ & $M L I$ & $C P$ & $S D E$ & $M L I$ & $C P$ & $S D E$ & $M L I$ & $C P$ & $S D E$ \\
\hline \multirow[t]{24}{*}{40} & 1 & 20 & 2 & 1 & 1.8566 & 0.946 & 0.0071 & 1.9125 & 0.959 & 0.0063 & 1.7564 & 0.912 & 0.0090 \\
\hline & & & & 2 & 0.9881 & 0.950 & 0.0069 & 1.0159 & 0.959 & 0.0063 & 0.8785 & 0.919 & 0.0086 \\
\hline & & & & 3 & 0.6935 & 0.965 & 0.0058 & 0.7046 & 0.967 & 0.0056 & 0.5874 & 0.921 & 0.0085 \\
\hline & & & 3 & 1 & 1.9761 & 0.977 & 0.0047 & 2.0458 & 0.979 & 0.0045 & 2.6347 & 0.912 & 0.0090 \\
\hline & & & & 2 & 1.3396 & 0.959 & 0.0063 & 1.3743 & 0.964 & 0.0059 & 1.3177 & 0.919 & 0.0086 \\
\hline & & & & 3 & 1.0236 & 0.966 & 0.0057 & 1.0390 & 0.968 & 0.0056 & 0.8812 & 0.921 & 0.0085 \\
\hline & & 30 & 2 & 1 & 1.5611 & 0.950 & 0.0069 & 1.5914 & 0.954 & 0.0066 & 1.4479 & 0.923 & 0.0084 \\
\hline & & & & 2 & 0.8009 & 0.962 & 0.0060 & 0.8143 & 0.964 & 0.0059 & 0.7249 & 0.925 & 0.0083 \\
\hline & & & & 3 & 0.5815 & 0.975 & 0.0049 & 0.5863 & 0.976 & 0.0048 & 0.4844 & 0.928 & 0.0082 \\
\hline & & & 3 & 1 & 1.7687 & 0.968 & 0.0056 & 1.8112 & 0.969 & 0.0055 & 2.1718 & 0.923 & 0.0084 \\
\hline & & & & 2 & 1.1540 & 0.963 & 0.0060 & 1.1728 & 0.965 & 0.0058 & 1.0873 & 0.925 & 0.0083 \\
\hline & & & & 3 & 0.8686 & 0.975 & 0.0049 & 0.8756 & 0.976 & 0.0048 & 0.7267 & 0.928 & 0.0082 \\
\hline & 2 & 20 & 2 & 1 & 1.5020 & 0.963 & 0.0060 & 1.6614 & 0.959 & 0.0063 & 1.7028 & 0.926 & 0.0083 \\
\hline & & & & 2 & 0.8449 & 0.955 & 0.0066 & 0.9115 & 0.957 & 0.0064 & 0.8388 & 0.928 & 0.0082 \\
\hline & & & & 3 & 0.6021 & 0.979 & 0.0045 & 0.6006 & 0.980 & 0.0044 & 0.5579 & 0.929 & 0.0081 \\
\hline & & & 3 & 1 & 1.5945 & 0.984 & 0.0040 & 1.6937 & 0.972 & 0.0052 & 2.5542 & 0.926 & 0.0083 \\
\hline & & & & 2 & 0.9852 & 0.988 & 0.0034 & 1.0367 & 0.985 & 0.0038 & 1.2582 & 0.928 & 0.0082 \\
\hline & & & & 3 & 0.7986 & 0.961 & 0.0061 & 0.8070 & 0.981 & 0.0043 & 0.8369 & 0.929 & 0.0081 \\
\hline & & 30 & 2 & 1 & 1.4057 & 0.941 & 0.0075 & 1.4119 & 0.945 & 0.0072 & 1.3959 & 0.928 & 0.0082 \\
\hline & & & & 2 & 0.7310 & 0.945 & 0.0072 & 0.7444 & 0.950 & 0.0069 & 0.6892 & 0.932 & 0.0080 \\
\hline & & & & 3 & 0.4610 & 0.0047 & 0.0047 & 0.4688 & 0.978 & 0.0046 & 0.4583 & 0.933 & 0.0079 \\
\hline & & & 3 & 1 & 1.4301 & 0.987 & 0.0036 & 1.4400 & 0.983 & 0.0041 & 2.0939 & 0.928 & 0.0082 \\
\hline & & & & 2 & 0.8427 & 0.992 & 0.0028 & 0.8723 & 0.990 & 0.0031 & 1.0338 & 0.932 & 0.0080 \\
\hline & & & & 3 & 0.6541 & 0.982 & 0.0042 & 0.6661 & 0.982 & 0.0042 & 0.6875 & 0.933 & 0.0079 \\
\hline
\end{tabular}


Table 5: The Lower (LL) and the Upper limits (UL), Maximum likelihood estimates (MLE), and the lengths of the 95\% confidence intervals (CI) for the parameters for $\alpha$ and $\beta$ using the kernel and classical approaches based on GOS based on the ball bearing data.

\begin{tabular}{|c|c|c|c|c|c|c|c|}
\hline Par. & $S$ & $n$ & $r$ & $\lambda$ & Inform Prior & Non Inform Prior & Classical \\
\hline \multirow{5}{*}{$\alpha$} & \multirow{3}{*}{$\begin{array}{l}\text { Type-II } \\
\text { censored } \\
\text { sample }\end{array}$} & \multirow[t]{3}{*}{23} & 11 & $\begin{array}{l}1 \\
2\end{array}$ & $\begin{array}{l}1.835 \text { (2.1832) } 4.018 \\
1.193(1.7667) 2.959\end{array}$ & $\begin{array}{cc}1.6888 & (2.3222) 4.011 \\
1.047 & (1.8747) 2.922\end{array}$ & $\begin{array}{cc}2.4166(4.8487) 7.265 \\
1.528 \quad(2.8469) 4.375\end{array}$ \\
\hline & & & 17 & $\begin{array}{l}1 \\
2\end{array}$ & $\begin{array}{cc}1.193 & (1.7677) 2.959 \\
0.977 & (1.1645) 2.142\end{array}$ & $\begin{array}{cc}1.377 & (1.8572) \\
0.234 \\
0.903 & (1.1631) 2.0663\end{array}$ & $\begin{array}{c}1.9985 \text { (2.3036) } 4.301 \\
1.345 \text { (1.4909) } 2.836\end{array}$ \\
\hline & & & 23 & $\begin{array}{l}1 \\
2\end{array}$ & $\begin{array}{l}1.491(1.2739) 2.765 \\
1.0368(0.857) 1.893\end{array}$ & $\begin{array}{l}1.436(1.2841) 2.7203 \\
0.9904(0.8608) 1.851\end{array}$ & $\begin{array}{ll}1.458 & (1.2885) \\
1.7446 \\
1.008 & (0.8607) 1.868\end{array}$ \\
\hline & $\begin{array}{l}\text { Type-II } \\
\text { progressive }\end{array}$ & & 11 & 2 & $\begin{array}{l}2.221(1.8228) 4.044 \\
1.604(1.7132) 3.318\end{array}$ & $\begin{array}{ll}2.1129 & (1.9294) \\
1.4735 & 4.042 \\
1.8351) & 3.3086\end{array}$ & $\begin{array}{ll}1.650 & (4.1296) 5.779 \\
1.0276 & (2.3151) 3.343\end{array}$ \\
\hline & samnle & & 17 & $\begin{array}{l}1 \\
2\end{array}$ & $\begin{array}{l}1.973(1.3614) 3.334 \\
1.395(1.4451) 2.840\end{array}$ & 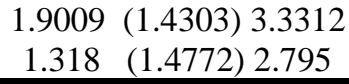 & $\begin{array}{ll}1.362 & (1.8615) 3.224 \\
0.9077 & (1.1570) \\
2.065\end{array}$ \\
\hline & & 23 & 11 & 1 & $\begin{array}{l}55.619 \text { (34.001) } 89.619 \\
35.601 \text { (21.291) } 56.892\end{array}$ & $\begin{array}{l}55.690(39.016) 94.706 \\
34.838 \text { (21.959) } 56.796\end{array}$ & $\begin{array}{l}51.435 \text { (23.016) } 74.451 \\
36.737(17.158) 53.895\end{array}$ \\
\hline & Type-II & & 17 & $\begin{array}{l}1 \\
2\end{array}$ & $\begin{array}{c}66.041(39.303) 105.34 \\
34.413(27.371) 61.784\end{array}$ & $\begin{array}{l}65.597(41.216) 106.81 \\
32.669(28.550) 61.219\end{array}$ & $\begin{array}{l}62.961 \text { (32.954) } 95.958 \\
34.231(25.777) 60.008\end{array}$ \\
\hline & sample & & 23 & 1 & $\begin{array}{l}66.208 \text { (36.018) } 102.23 \\
33.913 \text { (26.622) } 60.535\end{array}$ & $\begin{array}{r}65.467(36.794) \\
32.645(27.198) 59.26 \\
32.843 \\
\end{array}$ & $\begin{array}{l}65.022(33.714) 98.735 \\
33.857(26.253) 60.111 \\
\end{array}$ \\
\hline $\boldsymbol{B}$ & $\begin{array}{l}\text { Type-II } \\
\text { progressive }\end{array}$ & 23 & 11 & $\begin{array}{l}1 \\
2\end{array}$ & $\begin{array}{l}33.259 \text { (32.777)66.036 } \\
22.475 \text { (17.065) } 39.540\end{array}$ & $\begin{array}{ll}33.026 & (33.410) 66.436 \\
21.621 & (17.805) \\
39.426\end{array}$ & $\begin{array}{l}41.043(11.751) 52.794 \\
28.840(14.066) 42.906\end{array}$ \\
\hline & sample & & 17 & $\begin{array}{l}1 \\
2\end{array}$ & $\begin{array}{ll}45.481 & (32.434) \\
29.827 & 77.915 \\
(18.495) & 48.322\end{array}$ & $\begin{array}{l}45.315 \text { (32.749) } 78.064 \\
29.006(19.148) 48.154\end{array}$ & $\begin{array}{l}51.087 \text { (19.117) } 70.204 \\
32.311 \text { (18.217) 50.530 }\end{array}$ \\
\hline
\end{tabular}


Bayesian Inference on the Generalized Gamma Distribution

\section{References}

1. E. Stacy, A generalization of the gamma distribution. Annals of Mathematical Statistics, (1962) 1187-1192.

2. E. Stacy and G. Mihram, Parameter estimation for a generalized gamma distribution. Technometrics. 7 (1965) 349-358.

3. H. L. Harter, Maximum likelihood estimation of the parameters of a four parameters generalized gamma population from complete and censored samples. Technometrics. 9 (1967) 159-5.

4. H. Hager and L. Bain, Inferential procedures for the generalized gamma distribution. Journal of the American Statistical Association, 65, (1970), 1601-1609.

5. R. Prentice, A log gamma model and its maximum likelihood estimation. Biometrika. 61 (1974), 539-544.

6. J. Lawless, Inference on the generalized gamma and log gamma distributions. Technometrics, 22 (1980), 409-419.

7. J. Lawless, Statistical Models and Methods for Lifetime Data. John Wiley and Sons, New York, (1982).

8. T. Di Ciccio, Approximate inference for the generalized gamma distribution. Technometrics, (1987) 33-40.

9. D. R. Wingo, Computing maximum-likelihood parameter estimates of the generalized gamma distribution by numerical root isolation. IEEE Transactions on Reliability, 36 (1987) 586-590

10. J. Y. Wong, Readily obtaining the maximum-likelihood estimates of the 3- parameter generalized gamma-distribution of Stacy and Mirham. Microelectronics and Reliability, 33 (1993) 2243-2251.

11. A. C. Cohen and B. J. Whitten, Parameter Estimation in Reliability and Life Span Models. (Dekker, New York,( 1988).

12. M. S. Maswadah, Two approaches based on the structural model to inference on the generalized Gamma parameters. Egyptian Statist. J., 33(1) (1989) 110-129.

13. M. S. Maswadah, Structural inference on the generalized Gamma distribution based on type-II progressively censored sample. J. Austral. Math. Soc. Ser. A, 50(1) (1991) 15-22.

14 T. Y. Hwang and C. Y. Hu, On a characterization of the gamma distribution: the independence of the sample mean and the sample coefficient of variation. Annals of the Institute of Statistics Mathematics, 51, (1999) 749-753.

15. T. Hwang and P. Huang, On new moment estimation of parameters of the Generalized Gamma distribution and its characterization. Taiwanese journal of Mathematics, 10(4) (2006) 1083-1093.

16. O. Gomes, C. Combes and A. Dussauchoy, (2008). Parameter estimation of the generalized gamma distribution. Mathematics and Computers in Simulation,79(2008) 955-963.

17. C. Geng and L. Yuhiong, A Note on the Maximum Likelihood Estimation for the Generalized Gamma Distribution Parameters under Progressive Type-II 
Censoring. International Journal of Intelligent Technology and Applied Statistics, 2(2)(2009) , PP. 57-64.

18. A. Dadpay, E. Soofi and R. Soyer, Information measures for generalized gamma family. Journal of Econometrics, 56 (2007) 568-585.

19. B. Mukherjee, A. Gupta and S. K. Upadhyay, A Bayesian study for the comparison of generalized gamma model with its components. Sankhya B, 72 (2011), 154-174.

20. U. Kamps, A concept of generalized order statistics. Germany: B. G. Teubner Stuttgart, (1995).

21. M. Ahsanullah, The generalized order statistics and a characteristic property of the exponential distribution. Pak. J. Statist., 11(3) (1995) 215-218.

22. M. Ahsanullah, Generalized order statistics from exponential distribution. Journal of Statistical Planning and Inference, 85 (2000) 85-91.

23. G. E. P. Box and G. C. Taio, Bayesian inference in Statistical analysis, Addison Welsey (1973). 